\title{
USP7 is a novel Deubiquitinase sustaining PLK1 protein stability and regulating chromosome alignment in mitosis
}

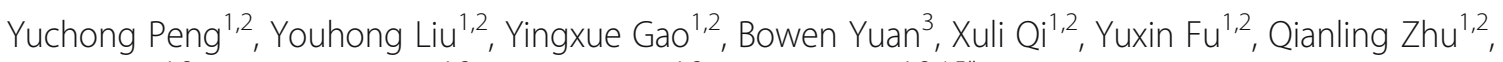
Tuoyu Cao ${ }^{1,2}$, Songwei Zhang ${ }^{1,2}$, Linglong Yin ${ }^{1,2}$ and Xiong Li Li, $^{1,4,5^{*}}$

\begin{abstract}
Background: The deubiquitinase USP7 has been identified as an oncogene with key roles in tumorigenesis and therapeutic resistance for a series of cancer types. Recently small molecular inhibitors have been developed to target USP7. However, the anticancer mechanism of USP7 inhibitors is still elusive.

Methods: Cell viability or clonogenicity was tested by violet crystal assay. Cell apoptosis or cell cycle was analyzed by flow cytometry, and chromosome misalignment was observed by a fluorescent microscopy. The protein interaction of PLK1 and USP7 was detected by tandem affinity purification and high throughput proteomics, and further confirmed by co-immunoprecipitation, GST pull-down and protein co-localization. The correlation between USP7 level of tumor tissues and taxane-resistance was evaluated.

Results: Pharmacological USP7 inhibition by P5091 retarded cell proliferation and induced cell apoptosis. Further studies showed that P5091 induced cell cycle arrest at G2/M phase, and particularly induced chromosome misalignment, indicating the key roles of USP7 in mitosis. USP7 protein was detected in the PLK1-interacted protein complex. USP7 interacts with PLK1 protein through its PBD domain by catalytic activity. USP7 as a deubiquitinase sustained PLK1 protein stability via the C223 site, and inversely, USP7 inhibition by P5091 promoted the protein degradation of PLK1 through the ubiquitination-proteasome pathway. By overexpressing PLK1, USP7 that had been depleted by RNAi ceased to induce chromosome misalignment in mitosis and again supported cell proliferation and cell survival. Both USP7 and PLK1 were overexpressed in taxane-resistant cancer cells, and negatively correlated with the MP scores in tumor tissues. Either USP7 or PLK1 knockdown by RNAi significantly sensitized taxaneresistant cells to taxane cell killing.

Conclusion: This is the first report that PLK1 is a novel substrate of USP7 deubiquitinase, and that USP7 sustained the protein stability of PLK1. USP7 inhibition induces cell apoptosis and cell cycle G2/M arrest, and overcomes taxane resistance by inducing the protein degradation of PLK1, resulting in chromosome misalignment in mitosis.
\end{abstract}

Keywords: USP7, PLK1, Chromosome misalignment, Cell cycle arrest, Apoptosis

\footnotetext{
* Correspondence: lixiongxiangya@csu.edu.cn

${ }^{1}$ Center for Molecular Medicine, Xiangya Hospital, Central South University,

Changsha, China

${ }^{2}$ Hunan Key Laboratory of Molecular Radiation Oncology, Xiangya Hospital,

Central South University, Changsha, China

Full list of author information is available at the end of the article
}

(c) The Author(s). 2019 Open Access This article is distributed under the terms of the Creative Commons Attribution 4.0 International License (http://creativecommons.org/licenses/by/4.0/), which permits unrestricted use, distribution, and reproduction in any medium, provided you give appropriate credit to the original author(s) and the source, provide a link to the Creative Commons license, and indicate if changes were made. The Creative Commons Public Domain Dedication waiver (http://creativecommons.org/publicdomain/zero/1.0/) applies to the data made available in this article, unless otherwise stated. 


\section{Background}

Protein stability is critical for normal cellular homeostasis. In addition to the autophagy-lysosome system, the ubiquitin-proteasome system (UPS) takes up approximately 80 to $90 \%$ of intracellular protein degradation [1]. In UPS-induced protein degradation, ubiquitin binds to target proteins and catalyzes them by a hierarchical cascade comprising E1, E2 and E3 ubiquitin ligases [2]. Inversely, the ubiquitination is removed from the labeled proteins or from polyubiquitin chains by deubiquitinating enzymes (or deubiquitinases, DUBs). DUBs are critical in cellular growth, survival and homeostasis, and are responsible for the turnover, localization and activity of their substrate proteins. Aberrant DUB activity results in a series of diseases, including cancer $[3,4]$.

Ubiquitin-specific proteases (USPs) are the largest DUB in all subfamilies, of which USP7 is the most prominent and well characterized member [5]. USP7 was originally identified as a binding partner for the herpes simplex virus (HSV) infected cell protein and named herpes-associated ubiquitin-specific protease (HAUSP) [6]. USP7 plays an important role in the cancer-related p53-MDM2 network [7-9]. USP7 specifically dequbiquitinates and stabilizes both p53 and MDM2 to various degrees, and USP7 inhibition is expected to inactivate MDM2 and activate p53, thereby leading to cell cycle arrest or apoptosis in cancer cells with functional p53 signaling [10]. In addition, USP7 promotes cell proliferation by stabilizing $\mathrm{Ki}-67$ protein [11]. USP7 is also involved in other cancer-associated mechanisms such as DNA damage and repair [12], epigenetic regulation [13], human terminal erythoid differentiation [14] and immune responses by regulating other cancer-related targets such as N-Myc [15], FOXO, PTEN and Claspin [5, 16]. USP7 is the first USP recognized as one of the cancer therapeutic DUB targets due to its important roles in tumorigenesis, cancer metastasis and HIV progression [17]. Several small molecular inhibitors of USP7 have been developed and are being tested in clinical trials [18]. The available data suggest that USP7 inhibitors induce cell cycle arrest and apoptosis in cancer cells through the p53 pathway, and sensitize cancer cells to PARP inhibitor-induced cell death [18]. P5091, a selective USP7 inhibitor, induces cell apoptosis by blocking the Wnt- $\beta$-catenin pathway [19]. Additionally, P5091 has an important role in anticancer immunity in the tumor microenvironment by inhibiting FOXP3 expression [20].

In addition to its roles in carcinogenesis, USP7 plays a critical role in therapeutic resistance. USP7-mediated MDC1 stabilization promotes cervical cancer cell survival and conferred cellular resistance to genotoxic insults [21]. USP7 knockdown overcomes Bortezomib resistance by suppressing the NF-kB signaling pathway in multiple myeloma [22]. USP7 inhibitors show great efficacy for inhibiting myeloma cell growth and overcoming NEK2-induced and acquired drug resistance in xenograft myeloma mouse models [23]. USP7 inhibition sensitizes p53-defective, chemotherapy-resistant chronic lymphoblastic leukemia (CLL) cells to clinically achievable doses of homologous recombination repair (HRR)-inducing chemotherapeutic agents in vitro and in vivo in a murine xenograft model [24]. Mitotic aberrance induces cell cycle arrest and apoptosis. A large number of molecules have been identified to be involved in the regulation of mitosis. The roles of USP7 in the regulation of mitosis have not been reported.

PLK1 is a master mitotic regulator controlling a wide variety of processes during G2/M cell cycle progression, such as centrosome maturation, entry into mitosis, chromosome segregation and cytokinesis. In prophase, PLK1 shuttles to the kinetochores, helping condensed chromosomes to align in the metaphase plate. At the onset of anaphase, PLK1 localizes at the spindle midzone and later to the cytokinetic bridge in order to coordinate cytokinesis and cell abscission. PLK1 has strong clinical relevance. It is overexpressed in many cancer types, and the degree of intratumoral overexpression correlates with poor patient prognosis [25, 26]. PLK1 has been considered a bona fide cancer target, and PLK1 inhibition results in aberrance of mitotic progression. PLK1 inhibition prevents the formation of a bipolar spindle, thus preventing the proper alignment of chromosomes in the metaphase plate. PLK1 inhibitors have been developed to experimentally treat different cancer types. Furthermore, PLK1 hyper-activation contributes to cancer therapeutic resistance to chemotherapy and radiotherapy [27]. Inversely, PLK1 depletion re-sensitizes cancer cells to therapy, overcoming therapeutic resistance.

In the present study, we for the first time found that USP7 inhibition induced chromosome misalignment in mitosis, thereby inducing G2/M cell cycle arrest and apoptosis. The possible mechanism is that USP7 protein physically interacts with PLK1 protein, and USP7 depletion induces the protein degradation of PLK1, thereby resulting in mitotic aberrance. Overexpressing PLK1 after USP7 knockdown by RNAi blocked the ability to induce chromosome misalignment in mitosis, while USP7 inhibition by P5091 retarded cell proliferation and cell survival and overcame taxane resistance. These data suggest that USP7 inhibitors induce mitotic aberrance by regulating PLK1 function, and thus show great potential for cancer therapy. 


\section{Methods}

\section{Cell culture and viral infection}

DU145, VCaP and HEK293T cells were obtained from American Type Culture Collection (Manassas, VA, USA). Nasopharyngeal cancer (NPC) cell line CNE2 was purchased from the Cancer Research Institute of Central South University (Changsha, Hunan, China). The paclitaxel-resistant NPC cell subline CNE2-TR and docetaxel-resistant cell subline DU145-DR were established by intermittently exposing the parental cells to gradually increasing concentrations of paclitaxel or docetaxel. Cells were maintained in DMEM or 1640 medium supplemented with $10 \%$ fetal bovine serum (FBS), 1\% penicillin, and streptomycin. Stable cell lines expressing USP7 shRNA were generated by transfecting DU145 or VCaP cells with PLKO.1/PLKO.1-USP7 shRNA plasmids, and were selected by $1 \mu \mathrm{g} / \mathrm{ml}$ puromycin. All cells were cultured in a humidified incubator at $37^{\circ} \mathrm{C}$ and $5 \% \mathrm{CO}_{2}$.

\section{Plasmids and siRNA transfection}

pGEX-4 T-1-PLK1, pCMV-USP7-Flag, pCMV-PLK1Flag and pCMV-PLK1 plasmids were constructed by generating the cDNA fragments of PLK1 and USP7 by PCR, and then subcloning them into the described vectors. To construct the USP7 and PLK1 mutants, we generated the cDNA fragments of wild-type or mutated PLK1 and USP7 by PCR, and then subcloned them into the above vectors. pLKO.1-USP7 shRNA plasmids were generated by subcloning USP7 shRNA into pLKO.1 lentiviral vector. (USP7 shRNA Sense: $5^{\prime}$-TGTATCTATT GACTGCCCTTT-3').

siRNA transfection was performed using a Mirus transfection kit according to the manufacturer's instructions (Mirus, Madison, WI, USA). The sequences of USP7 siRNA $\left(5^{\prime}-3^{\prime}\right)$ are as follows: USP7\#1 (UGUAUCUAUU GACUGCCCU), USP7\#2 (CGUGGUGUCAAGGUGU ACU) and PLK1 (CACCAUAUGAAUUGUACAG).

\section{RNA extraction and real-time quantitative PCR}

Total RNA was isolated using the RNAiso Plus method following the manufacturer's protocol (Takara Bio Inc., Beijing, China). The concentration and quality of dissolved RNA samples was determined by a spectrophotometer before the conversion to cDNA. Quantitative real-time PCR was then performed using a real-time PCR detection system (Thermo Fisher Scientific 7500) according to the manufacturer's instructions (Waltham, MA, USA).

\section{Antibodies and reagents}

Antibodies and reagents used in the study and their sources are listed as follows: anti-USP7, anti-PLK1, antiUbiquitin, anti- $\alpha$-tubulin, mouse IgG were purchased from Cell Signal Transduction (CST, Danvers, MA, USA). Anti- $\beta$-actin antibody was purchased from Santa Cruz Biotechnology (Dallas, UT, USA), anti-Flag was purchased from Sigma Aldrich (St. Louis, MO, USA) and rabbit IgG was purchased from Protein-tech (Wuhan, China). Anti-USP7 antibody for immunohistochemistry was purchased from ABclonal Technology (Upper Heyford, UK). The USP7 inhibitor P5091 was purchased from MedChemExpress (MCE, Shanghai, China), CHX was purchased from CST, and MG132 was purchased from Selleck Chemicals (Houston, Texas, USA).

\section{Immunoblotting and immunoprecipitation}

Cells were lysed and protein was extracted using RIPA buffer supplemented with protease inhibitor cocktails (Selleck Chemicals). Protein concentration was measured with a Bradford assay kit (Thermo Fisher Scientific). An equal amount of protein was loaded and separated by SDS-polyacrylamide gel electrophoresis (SDS-PAGE) with $\beta$-actin as the loading control.

Extracts for immunoprecipitation were prepared using RIPA buffer supplemented with protease inhibitor cocktails (Selleck Chemicals). The extracts were then incubated with the indicated antibodies at $4{ }^{\circ} \mathrm{C}$ for $12 \mathrm{~h}$ on a rotator, followed by incubation with proteinA/G-magnetic beads (MCE) at $4{ }^{\circ} \mathrm{C}$ for $1 \mathrm{~h}$ on a rotator. After incubation, beads were washed four times in immunoprecipitation buffer and boiled in $1 \times$ loading buffer. Protein samples were analyzed by SDS-PAGE.

\section{GST pull-down assay}

pGEX-4 T-1-PLK1 was constructed using the GST gene fusion system according to the manufacturer's instructions. To produce glutathione S-transferase (GST) and GST-PLK1 fusion proteins, pGEX-4 T-1 and pGEX-4 T1-PLK1 were individually transfected into BL21 complete cells. Protein expression was induced by ispropyl b-D-1-thiogalactopyranoside (IPTG). GST-PLK1 and GST proteins were purified by binding to glutathionesepharose resins (Sangon Biotech, Shanghai, China). Resins were incubated with cell lysates from DU145 cells at $4{ }^{\circ} \mathrm{C}$ for $12 \mathrm{~h}$ on a rotator, and then were washed with the washing buffer four times. Resin-bound complexes were eluted by boiling, and subjected to western blotting.

\section{Cellular immunofluorescence}

Cells grown on coverslips were fixed with $4 \%$ paraformaldehyde solution for $15 \mathrm{~min}$ at room temperature, permeabilized with $0.25 \%$ Triton X-100 for $20 \mathrm{~min}$, and then blocked with $5 \%$ bovine serum albumin for $1 \mathrm{~h}$. Cells were incubated with the primary antibodies as indicated at $4{ }^{\circ} \mathrm{C}$ for $12 \mathrm{~h}$, and then incubated with the fluorescent-labeled secondary antibodies. The nuclei 

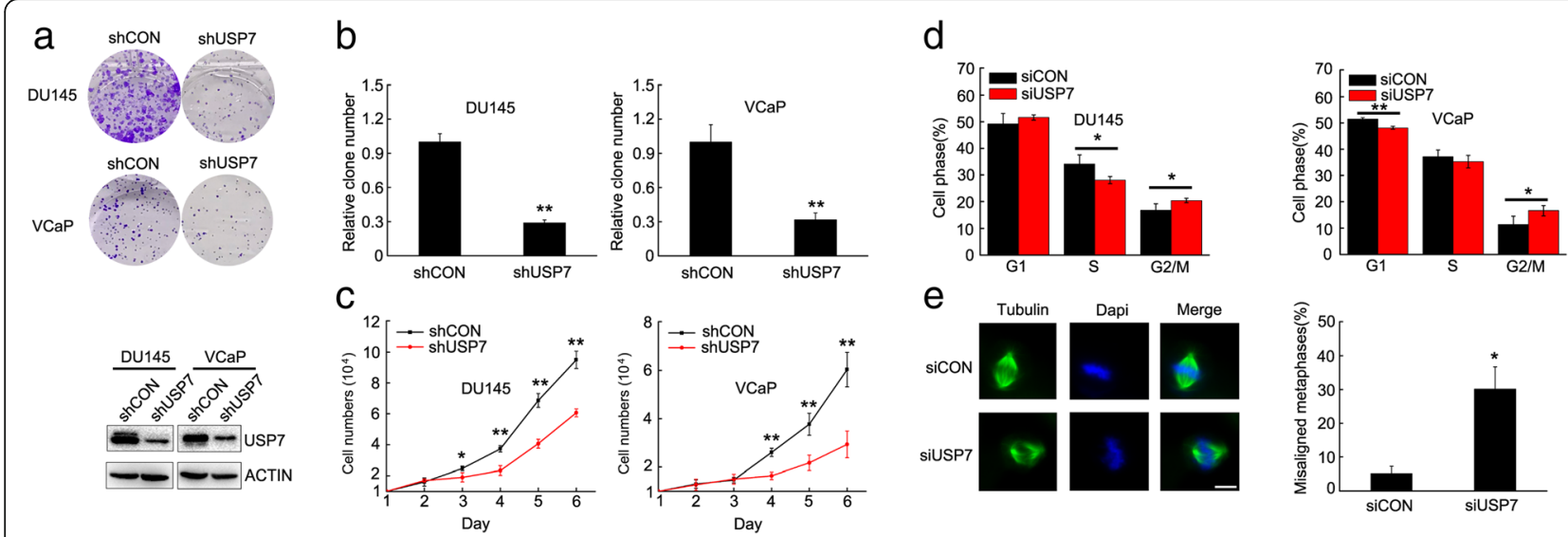

Fig. 1 Depletion of USP7 retarded cell proliferation by inducing G2/M cell cycle arrest and chromosome misalignment in mitosis. a and $\mathbf{b}$ DU145 or VCaP cells stably expressing USP7 or control shRNA were generated. The same number of cells were seeded and the colonies formed were counted 14 days later. The knockdown efficiency of USP7 was tested by western blot. c The growth of DU145 or VCaP cells stably expressing USP7 or control shRNA were compared every day after cell seeding. Standard deviation bars were obtained from triplicate repeats. $\mathbf{d}$ The cell cycle distribution of DU145 or VCaP cells expressing USP7 or control siRNA were analyzed by flow cytometry. Standard deviation bars were obtained from three independent experiments. e In DU145 or VCaP cells expressing USP7 or control siRNA, spindles were stained with immunofluorescent a-tubulin antibody and nuclear DNA were stained with DAPI. The number of cells with misaligned chromosomes were quantified (scale bar, $10 \mu \mathrm{M}$ )

were stained with DAPI. Images were captured and analyzed with Zeiss fluorescence microscopy.

\section{Flow cytometry}

For cell cycle analysis, cells were first fixed in $70 \%$ ethanol at $4{ }^{\circ} \mathrm{C}$. DNA was stained with $0.02 \mathrm{mg} / \mathrm{ml}$ propidium iodide containing RNase $(30 \mu \mathrm{g} / \mathrm{ml})$ at $37^{\circ} \mathrm{C}$ for 30 min. Cell cycle phase distribution was analyzed by flow cytometry (Millipore, Temecula, CA, USA).

Apoptosis was detected with an Annexin V-FITC kit. Briefly, cells were incubated with $5 \mu$ l Annexin V-FITC and $5 \mu \mathrm{l}$ propidium iodide in $100 \mu \mathrm{l}$ binding buffer for $15 \mathrm{~min}$ at room temperature, immediately followed by flow cytometry analysis.

\section{MTS cell viability assay}

Cells were cultured in 96-well flat-bottomed microtiter plates (5000 per well), and treated with paclitaxel or docetaxel with or without P5091 for $24 \mathrm{~h}$. Then $20 \mu \mathrm{l}$ of MTS solution [3-(4,5-dimethylthiazol-2-yl)-5-(3-carboxymethoxyphenyl)-2-(4-sulfophenyl)-2Htetrazolium]

(Promega, Madison, WI, USA) was added to each well, and cultured at $37^{\circ} \mathrm{C}$ in $5 \% \mathrm{CO}_{2}$ for $2 \mathrm{~h}$. Absorbance at $490 \mathrm{~nm}$ was measured by spectrometer. Each treatment was performed in quintuplicate. The relative survival rate of the cancer cells was calculated according to the cell viability.

\section{Immunohistochemistry}

Paraffin sections of breast tumor tissues were obtained from the Department of Pathology, Xiangya Hospital, Central South University. The slides were incubated with primary antibody against USP7. For negative control, isotype-matched antibodies were applied. USP7 positive signals were detected in both nuclear and cytoplasmic compartments. The IHC results were judged from 5 to 10 random fields $(\times 400)$ by two independent senior pathologists. The degree of staining was classified into four stages (- negative, + slight yellow, ++ brown, +++ dark brown or $\tan )$. The ratio of positive cells was divided into three stages $(+<25 \%,++25$ to $49 \%,+++\geq 50 \%)$. The overall standard including staining degree and ratio of positive cells: + percent of positive cells $\times 1,++$ percent of positive cells $\times 2$ and +++ percent of positive cells $\times 3$. The overall results: $+<1,++1$ to $1.5,+++>1.5$.

\section{Statistical analysis}

All data were analyzed by Origin 8 . Results are presented as mean \pm SD. One-way ANOVA was used to analyze the statistical difference of multiple groups. ${ }^{*} P<0.05$ and ${ }^{* *} P<0.01 . P<0.05$ was considered as statistically significant.

\section{Results}

USP7 depletion impaired cell colony formation and retarded cell proliferation by inducing G2/M cell cycle arrest and chromosome misalignment in mitosis

USP7 has been identified as an oncogene, and plays a key role in tumorigenesis in several cancer types, including prostate cancer. We generated two cell lines, DU145 and $\mathrm{VCaP}$ with USP7 stable knockdown, by infecting cells with lentiviral vector expressing USP7 shRNA, and compared their ability to form cell colonies and proliferate. After USP7 knockdown the number of cell colonies 


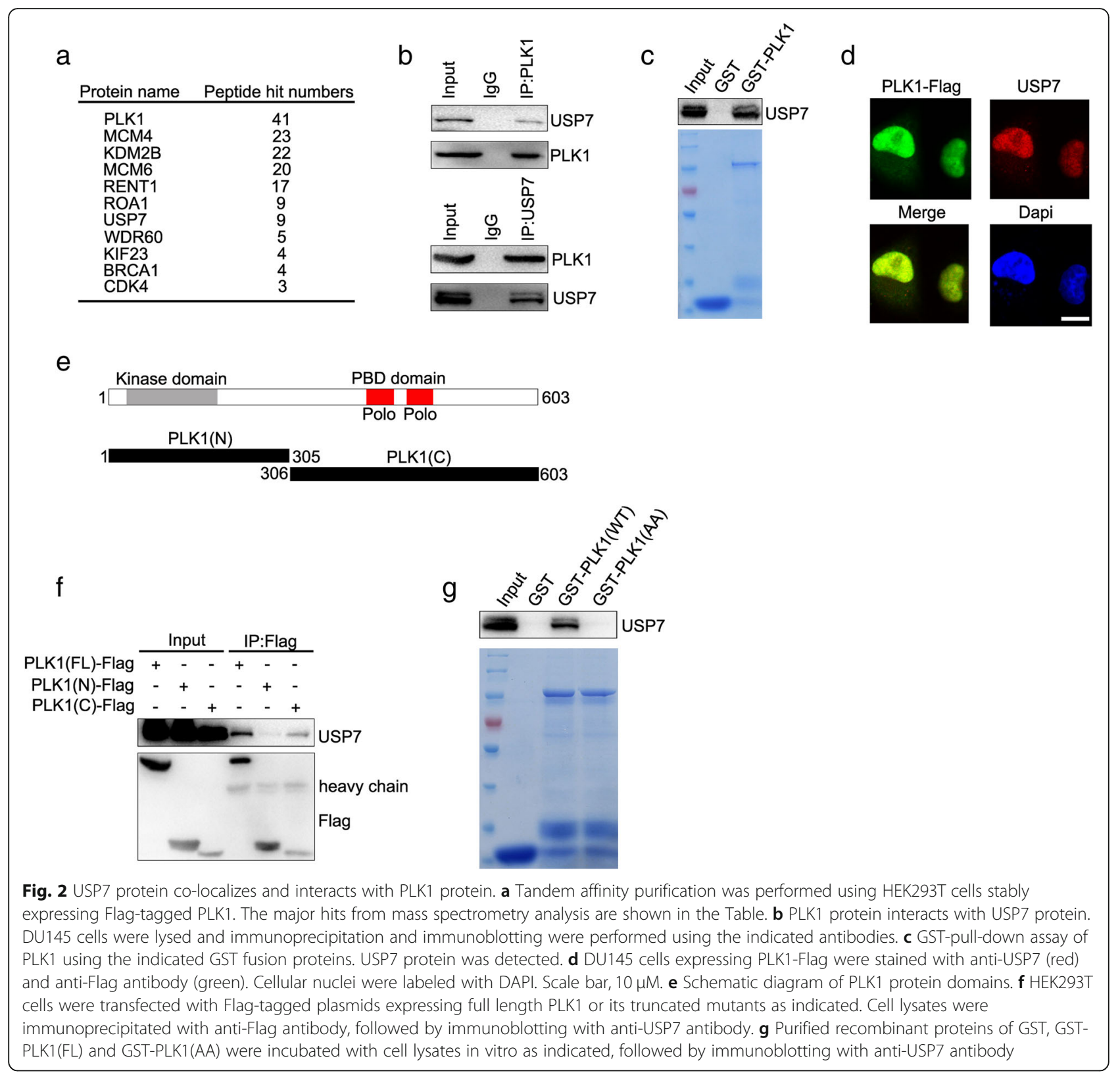

significantly decreased (Fig. 1a and b) and cell proliferation was significantly retarded (Fig. 1c).

To further explore the mechanisms by which USP7 depletion impaired cell colony formation and retarded cell proliferation, we tested changes of cell cycle distribution when USP7 was knockdown with siRNA. Consistently in the two cell lines, USP7 depletion significantly increased the cell population at G2/M phase, indicating cell cycle arrest (Fig. 1d). We further monitored chromosome alignment in mitosis when USP7 was knockdown with siRNA. USP7 knockdown significantly elevated the percentage of cells with misaligned chromosome in mitosis (Fig. 1e). These results indicated that USP7 is a critical molecule with a key role in mitosis.
PLK1 is a novel substrate of USP7, and USP7 sustains the protein stability of PLK1

In another ongoing research project, we screened PLK1associated proteins by tandem affinity purification with an anti-Flag antibody, followed by high throughput proteomics by using a mass spectrometer. Beyond our expectation, USP7 was identified as a novel substrate of PLK1 at the top of the list of PLK1-interactive proteins (Fig. 2a). The protein interaction of USP7 and PLK1 was validated by co-immunoprecipitation. USP7 protein was detected when PLK1 was immunoprecipitated by PLK1 antibody, and inversely PLK1 was detected when USP7 was immunoprecipitated in DU145 cells (Fig. 2b). We further revealed that USP7 protein physically interacts 


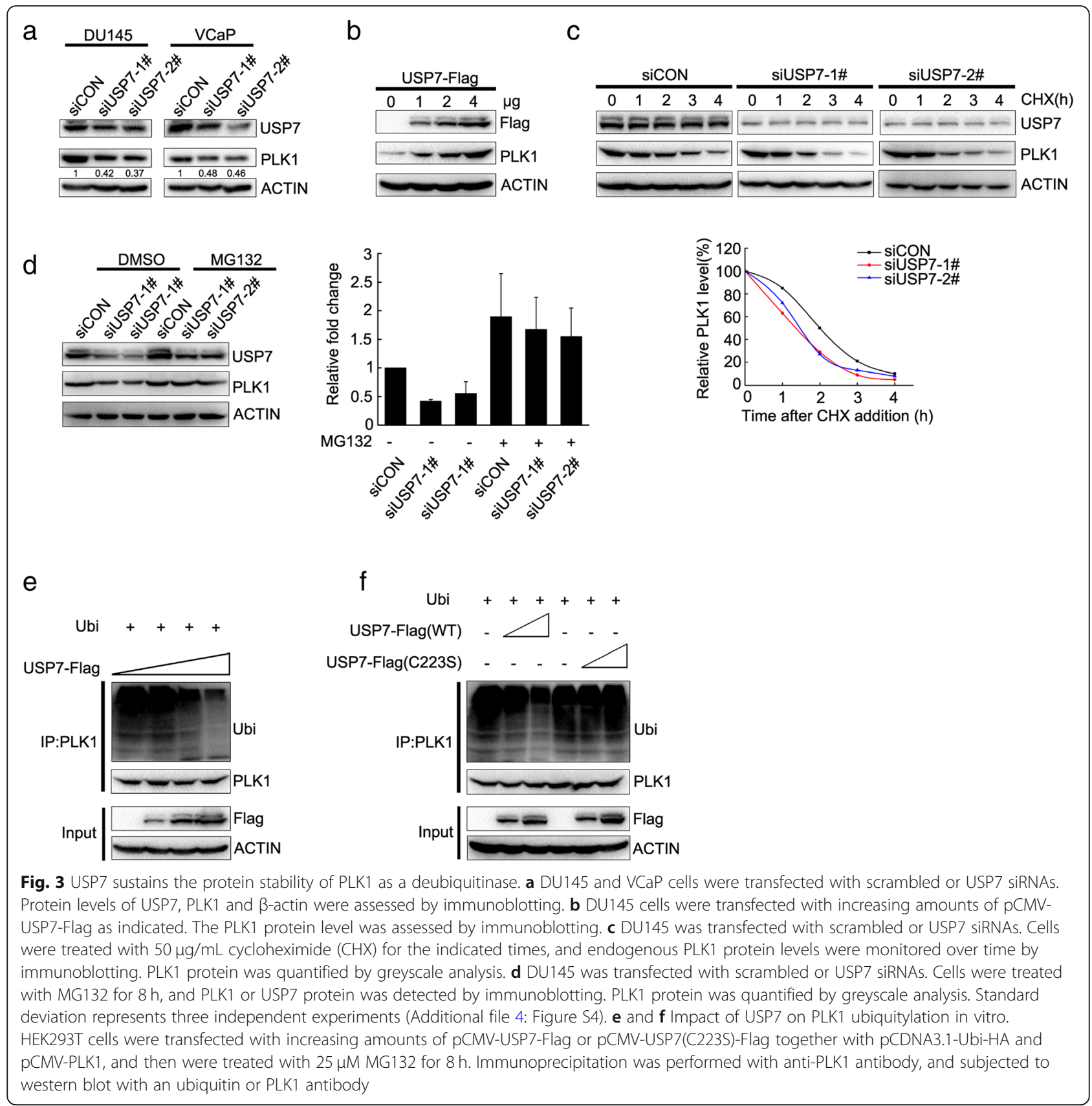

with PLK1 by GST pull down assay (Fig. 2c). The protein interaction of USP7 and PLK1 was further validated in DU145 cells by observing their intercellular colocalization (Fig. 2d). The structure of PLK1 protein is divided into a $\mathrm{N}$ terminal fragment containing a kinase domain and a $\mathrm{C}$ terminal fragment containing a PBD domain (Fig. 2e). Co-immunoprecipitation validated USP7 interaction with the $\mathrm{C}$ terminal domain of PLK1 protein (Fig. 2f). H538A/K540A (AA) mutations that disrupt the protein-interacting capability of PBD [28] result in the loss of PLK1 and USP7 protein interaction (Fig. 2g). The polo box motifs of PLK1 protein are critical for its protein interaction with USP7.

\section{USP7 sustains the protein stability of PLK1 as a deubiquitinase}

Since USP7 protein, as a deubiquitinase, physically interacts with PLK1 protein, we tested whether USP7 depletion influenced PLK1 protein stability. In both DU145 and VCaP cells, the depletion of USP7 significantly decreased PLK1 protein levels but no effect on PLK1 mRNA (Fig. 3a; Additional file 1: Figure S1A-B). Inversely, when USP7 


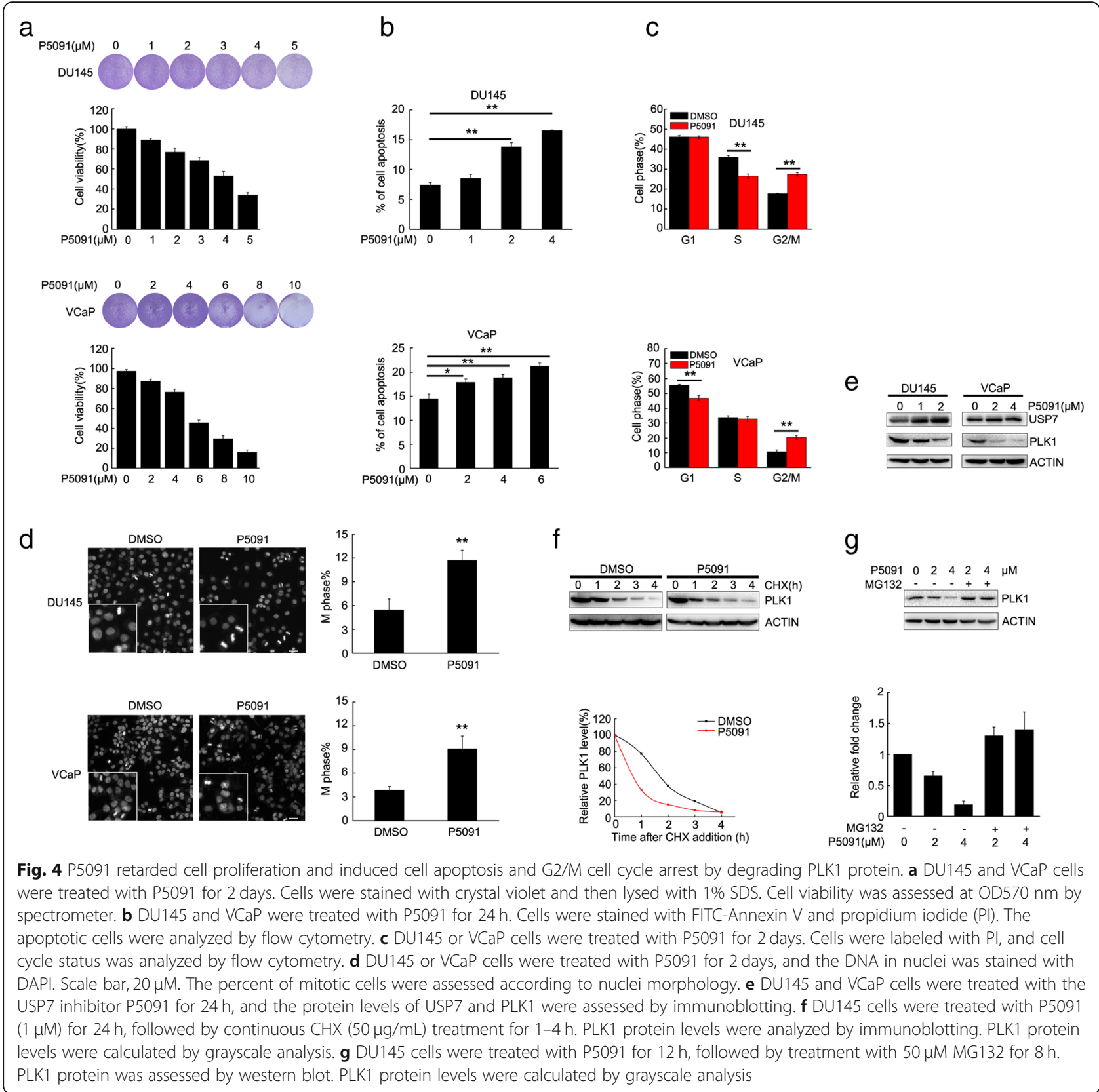

protein levels were elevated by transient transfection, the protein level of PLK1 accordingly rose in a dosedependent manner (Fig. 3b).

To explore the mechanism by which USP7 sustains PLK1 protein stability, we assessed the half-life of PLK1 protein when USP7 was depleted. The protein synthesis was inhibited by cycloheximide (CHX), and PLK1 protein was assessed at different time points. The knockdown of USP7 with siRNA promoted the protein degradation of PLK1 and shortened the half-life of PLK1 protein (Fig. 3c). The degradation of PLK1 protein was inhibited by MG132, an inhibitor of the ubiquitination-proteasome pathway, indicating that USP7 sustained PLK1 protein stability through the ubiquitination-proteasome pathway (Fig. 3d).

We further validated the results by co-transfecting the plasmids expressing HA-ubiquitin or PLK1 to HEK293T cells. PLK1 protein was immunoprecipitated, and the ubiquitinated protein was detected with ubiquitin antibody. The ubiquitin protein steadily decreased with the increase of USP7 (Fig. 3e). USP7 is a deubiquitinase, and C223 has been identified as the catalytic site for its deubiquitination function [29]. When C223 was mutated, the elevation of USP7 protein levels did not decrease PLK ubiquitinated protein levels (Fig. 3f). The results further validated that 


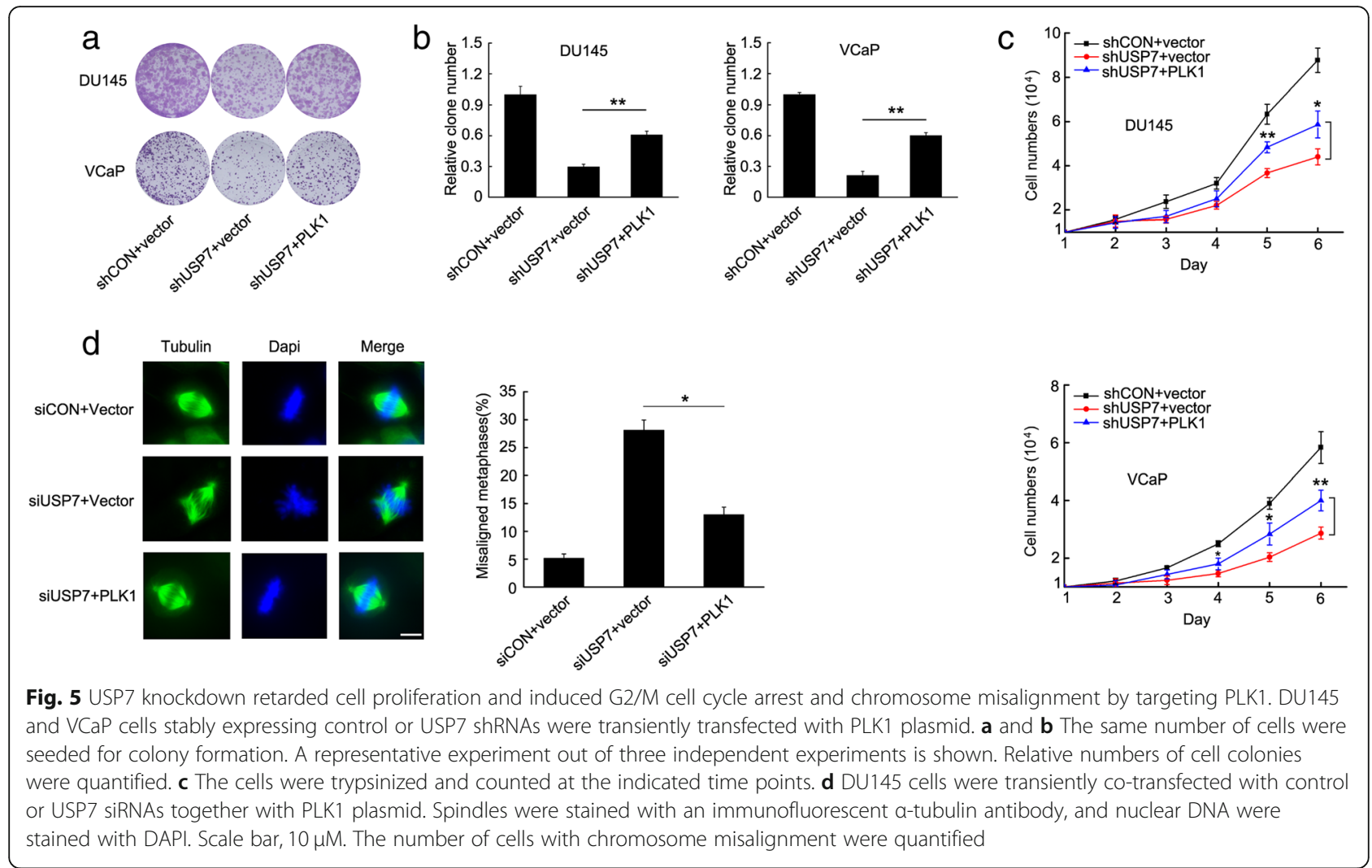

USP7 sustains PLK1 protein stability based on the deubiquitination catalytic activity of USP7.

The USP7 inhibitor P5091 retarded cell proliferation and induced cell apoptosis and G2/M cell cycle arrest

P5091, a selective USP7 inhibitor, has shown potential anticancer efficacy in pre-clinical trials [30, 31]. We tested the impact of P5091 on the viability of prostate cancer DU145 and VCaP cells. Consistently in these two cell lines, P5091 significantly decreased cell viability in a dose-dependent manner (Fig. 4a). We investigated whether P5091 decreased cell viability by inducing cell apoptosis and cell cycle arrest. P5091 induced cell apoptosis in a dose-dependent manner (Fig. 4b), and also induced G2/M cell cycle arrest (Fig. 4c). We further clarified whether P5091 induced G2/M cell cycle arrest by increasing mitotic cells. As shown in Fig. 4d, P5091 induced more mitotic cells based on nuclear morphology. P5091 induced the protein degradation of PLK1 in a dose-dependent manner (Fig. 4e) and shortened the half-life of PLK1 protein, when new protein synthesis was inhibited by CHX (Fig. 4f). PLK1 protein degradation could be inhibited by MG132 (Fig. 4g), validating that USP7 sustains PLK1 protein through the ubiquitination-proteasome pathway. The biological effects of the USP7 inhibitor were very similar to USP7 knockdown with RNAi.
PLK1 rescued USP7 knockdown-induced cell viability, cell proliferation and chromosome misalignment

To test USP7's biological functions by regulating PLK1, we artificially elevated PLK1 when USP7 was knocked down by RNAi (Additional file 3: Figure S3A) and tested cell colony formation, cell proliferation and chromosome status. As expected, USP7 knockdown with shRNA significantly decreased cell colony formation and retarded cell proliferation, but PLK1 elevation rescued cell colony formation and cell proliferation (Fig. 5a, b, c). Furthermore, USP7 knockdown induced chromosome misalignment in mitosis, but artificial elevation of PLK1 restored chromosome alignment in mitosis (Fig. 5d). These results validated the critical role of USP7 in cell proliferation and cell cycle transition by regulating PLK1.

USP7 is positively correlated with PLK1, and contributes to taxane resistance

We tested the expression and intercellular localization of USP7 protein by immunohistochemical staining in tissue sections of primary breast cancer. The sensitivity of tumor tissues to taxane was evaluated by the MP (Miller-Payne) grading scale, a system for the evaluation of patient response to taxane chemotherapy. The expression of USP7 is very high in breast cancer sections with low MP scores, while USP7 levels are low in MP high breast cancer sections (Fig. 6a). Thus USP7 expression 
a

\begin{tabular}{ccc}
\hline Sample & MP & USP7 \\
\hline 1219465 & 1 & +++ \\
1216958 & 1 & +++ \\
1169195 & 1 & +++ \\
1173010 & 1 & ++ \\
1200483 & 1 & ++ \\
1175212 & 1 & ++ \\
1214116 & 2 & ++ \\
1210937 & 3 & +++ \\
1204481 & 3 & ++ \\
1215478 & 3 & ++ \\
1215125 & 3 & ++ \\
1219193 & 3 & + \\
1207642 & 3 & + \\
1207691 & 3 & + \\
1215776 & 3 & + \\
1216961 & 3 & + \\
1191452 & 3 & + \\
\hline
\end{tabular}

C
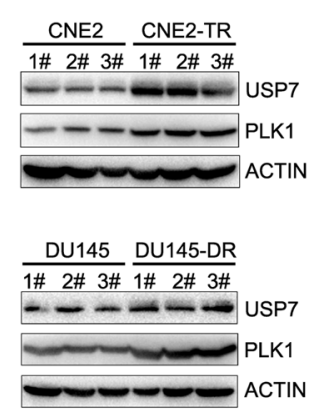

f

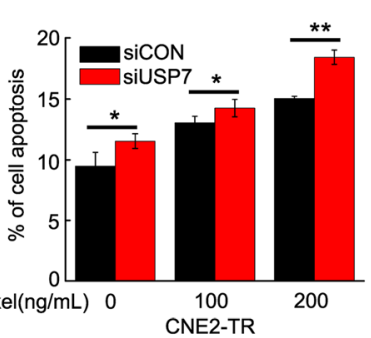

$\begin{array}{ccc}\text { Paclitaxel(ng/mL) } 0 & 100 \\ \text { CNE2-TR }\end{array}$

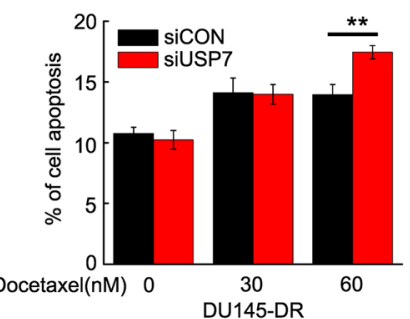

b
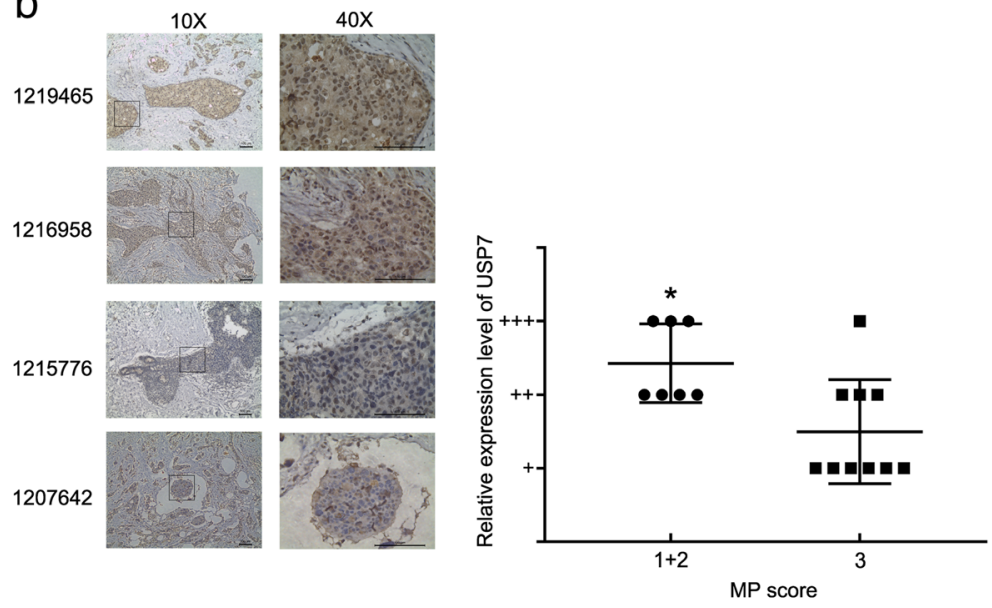

d

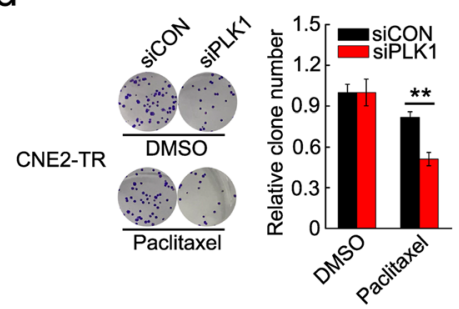

e
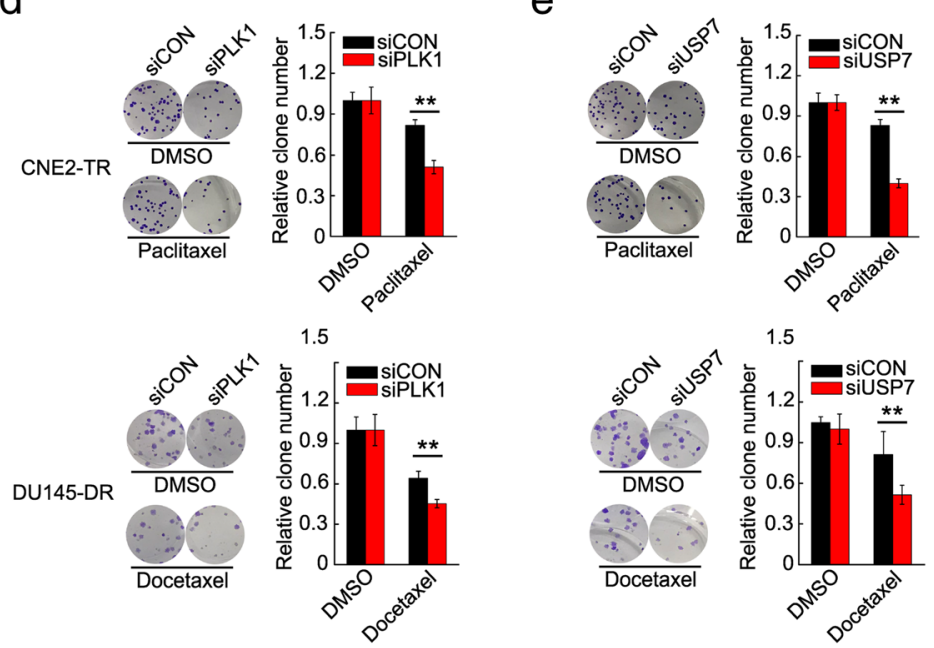

g

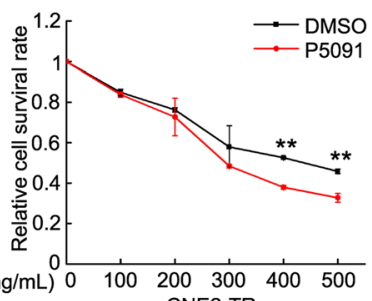

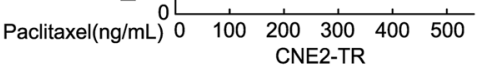

h
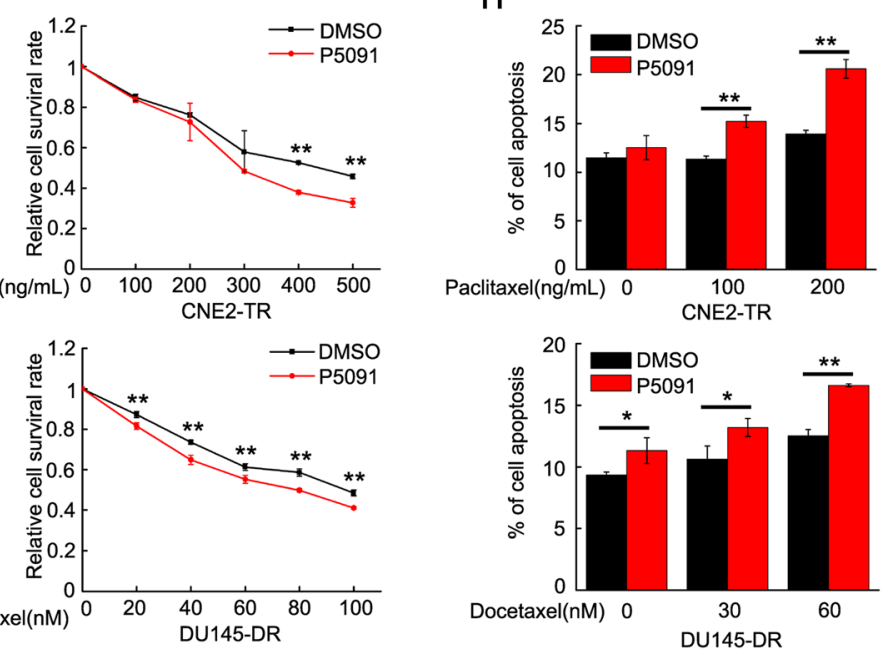

Fig. 6 (See legend on next page.) 
(See figure on previous page.)

Fig. 6 USP7 is positively correlated with PLK1, and contributes to taxane resistance. a Expression levels and localizations of USP7 protein were detected by IHC in breast cancer tissues. b Summary of USP7 IHC positive signal and MP (Miller-Payne) score in primary breast tumor sections. USP7 expression levels were negatively correlated with MP score by statistical analysis using the Mann-Whitney test. c Paclitaxel-resistant CNE2-TR or docetaxel-resistant DU145-DR cells were generated, and USP7 and PLK1 proteins were assessed by western blot. $\mathbf{d}$ and e PLK1 or USP7 were depleted with siRNA in CNE2-TR and DU145-DR cells, and the cells were treated with DMSO, paclitaxel $(100 \mathrm{ng} / \mathrm{mL})$ or docetaxel $(40 \mathrm{nM})$ for $48 \mathrm{~h}$. The same number of cells were seeded for colony formation. Relative numbers of cell colonies were quantified. $\mathbf{f}$ USP7 was depleted with siRNA in CNE2-TR or DU145-DR cells, and cells were treated with paclitaxel or docetaxel for $24 \mathrm{~h}$. The cells were stained with Annex-V/PI, and apoptotic cells were detected by flow cytometry. $\mathbf{g}$ CNE2-TR or DU145-DR cells were treated with or without P5091 (2 $\mu \mathrm{M})$ together with paclitaxel or docetaxel at the doses shown for $24 \mathrm{~h}$. Cell viability was tested by MTS assay. $\mathbf{h}$ CNE2-TR or DU145-DR cells were treated with or without P5091 $(2 \mu \mathrm{M})$ together with paclitaxel or docetaxel at the doses shown for $24 \mathrm{~h}$. The cells were stained with Annex-V/PI, and apoptotic cells were detected by flow cytometry

levels were negatively correlated with MP scores (Fig. 6b). The data further validated that USP7 may be a reliable biomarker of taxane resistance, in addition to a biomarker of cancer malignancy.

Paclitaxel or docetaxel is the first-line chemotherapeutic drug for prostate cancer and nasopharyngeal carcinoma patients. However, taxane resistance often develops after a period of treatment. We developed two taxane-resistant cell line, CNE2-TR and DU145-DR. The resistance of cells to taxane were assessed by cell viability assay (Additional file 2: Figure S2A-B) and apoptosis detectionassay (Additional file 2: Figure S2CD). We found higher expression of both USP7 and PLK1 proteins in docetaxel-resistant DU145-DR cells and paclitaxel-resistant CNE2-TR cells (Fig. 6c), and PLK ubiquitinated protein levels were decreased in taxane-resistant cells (Additional file 1: Figure S1C). The knockdown of USP7 or PLK1 in DU145-DR or CNE2-TR cells significantly weakened colonyformation ability (Fig. 6d, e). The knockdown efficiency of USP7 and PLK1 were tested by western blot (Additional file 3: Figure S3B-C). USP7 depletion also significantly increased the population of apoptotic cells in the two cell lines (Fig. 6f). Consistently, the USP7 inhibitor P5091 significantly retarded cell proliferation and increased the population of apoptotic cells in both DU145-DR and CNE2-TR cells (Fig. 6g, h).

\section{Discussion}

USP7 has been identified as an oncogene with an important role in carcinogenesis and therapeutic resistance in a variety of cancer types. USP7 has also been regarded as an effective anticancer target, and several small molecular inhibitors have been developed to target USP7. However, no DUB inhibitor has stepped up to clinical trials, though the USP7 inhibitor P5091 showed significant anti-cancer effects in pre-clinical tests. In our present study, USP7 inhibition by P5091 significantly retarded cell proliferation and reduced cell colony formation, and USP7 inhibition by P5091 induced G2/M cell cycle arrest and cell apoptosis.
The anti-cancer effect of USP7 knockdown is associated with interference in some cancer-associated pathways, such as p53-MDM2 axis, Ki-67, c-Myc, FOXO, PTEN and Claspin, which are critical for DNA damage repair, epigenetic regulation and immune responses $[18,32]$. Unexpectedly, we for the first time found that USP7 knockdown induced chromosome misalignment in mitosis. The exact molecular mechanism by which USP7 controls mitosis is currently unknown.

By using high-throughput proteomics methods, we detected USP7 protein in the protein complex immunoprecipitated by PLK1 antibody, and the interaction of USP7 and PLK1 was validated by co-immunoprecipitation. The PBD domain of PLK1, especially two polo motifs in the domain, interacts with USP7 protein. USP7 sustained PLK1 protein stability, and conversely USP7 inhibition by P5091 promoted PLK1 protein degradation through the ubiquitination-proteasome pathway. It is well known that PLK1 is a master mitotic regulator, and controls centrosome maturation, entry into mitosis, chromosome segregation and cytokinesis $[33,34]$. PLK1 inhibition results in aberrant mitotic progression. PLK1 inhibition prevents the formation of a bipolar spindle and misalignment of chromosomes in the metaphase plate. PLK1 inhibitors have been developed for cancer therapy. Overexpressing PLK1 reversed USP7 knockdown by RNAi and restored chromosome alignment in mitosis. These data validated that USP7 controls mitosis by regulating PLK1.

USP7 and PLK1 have strong clinical relevance, being overexpressed in tumor cells in a many cancer types. The degree of intratumoral overexpression closely correlates with poor patient prognosis and sensitivity to taxane chemotherapy (MP). Both USP7 and PLK1 have been considered bona fide cancer targets, and USP7 or PLK1 inhibitors have been developed to experimentally treat different cancer types or to re-sensitize cancer cells to overcome therapeutic resistance.

\section{Conclusion}

In this study we report for the first time that USP7 knockdown resulted in aberrant mitosis, and the effect 
was achieved via PLK1, a critical mitotic regulator. Furthermore, we report for the first time that PLK1 is a novel substrate of USP7 deubiquitinase, and that USP7 sustained the protein stability of PLK1. USP7 knockdown or inhibition induced cell apoptosis and cell cycle G2/M arrest and overcame taxane resistance by inducing the protein degradation of PLK1, resulting in chromosome misalignment in mitosis. The single or combined administration of USP7 or PLK1 inhibitors has demonstrated great anticancer potential for future clinical use.

\section{Supplementary information}

Supplementary information accompanies this paper at https://doi.org/10. 1186/s13046-019-1457-8.

Additional file 1. Figure S1. (A-B) DU145 and VCaP were transfected with a scramble or two different USP7 siRNAs for $48 \mathrm{~h}$.

Additional file 2. Figure S2. Assessment of drug resistance of paclitaxel-resistant and docetaxel-resistant cells.

Additional file 3. Figure S3. USP7 and PLK1 protein levels were evaluated by western blotting.

Additional file 4. Figure S4. The original blots

\section{Abbreviations}

CHX: Cycloheximide; CST: Cell Signaling Technology; DUB: Deubiquitinating enzymes; HAUSP: Herpes-associated ubiquitin-specific protease; HSV: Herpes simplex virus; MCE: MedChemExpress; MP: Miller-Payne; PBD: Polo-box domains; PLK1: Polo-like kinase 1; SD: Standard Deviation; UPS: Ubiquitin proteasome system; USP7: Ubiquitin specific protease 7; USPS: Ubiquitinspecific proteases

\section{Acknowledgements}

We thank the members of the Hunan Key Laboratory of Viral Hepatitis and Center of Molecular Medicine, Xiangya Hospital, Central South University for constructive discussion of this study.

\section{Authors' contributions}

PYC performed experiments, analyzed data and wrote the manuscript; LYH, GYX and YBW participated in the assays of cell viability and clongenicity; QXL and FXY participated in co-IP assay, ZQL, CTY and ZSW participated in apoptosis analysis and cell immunofluorescence; YLL participated in data analysis; LX designed the project, supervised all experiments, analyzed results, and wrote the manuscript. All authors read and approved the final manuscript.

\section{Funding}

This work was supported by grants from the National Natural Science Foundation of China (NSFC, 81572542 and NSFC, 81874196).

\section{Availability of data and materials}

The data that support the findings of this study are available from the corresponding authors upon reasonable request.

\section{Ethics approval and consent to participate}

No applicable.

\section{Consent for publication}

Not applicable.

\section{Competing interests}

All authors declare that they have no competing interests.

\section{Author details}

${ }^{1}$ Center for Molecular Medicine, Xiangya Hospital, Central South University, Changsha, China. ${ }^{2}$ Hunan Key Laboratory of Molecular Radiation Oncology, Xiangya Hospital, Central South University, Changsha, China. ${ }^{3}$ Department of
Pathology, The Third Xiangya Hospital, Central South University, Changsha, China. ${ }^{4}$ School of Clinical Pharmacy, Guangdong Pharmacology University, Guangzhou, China. ${ }^{5}$ The First Affiliated Hospital, Guangdong Pharmacology University, 19 Nonglinxia Road, Yuexiu District, Guangzhou, Guangdong,

China

Received: 2 September 2019 Accepted: 17 October 2019

Published online: 15 November 2019

\section{References}

1. Hershko A, Ciechanover A. The ubiquitin system for protein degradation. Annu Rev Biochem. 1992;61:761-807.

2. Behrends C, Harper JW. Constructing and decoding unconventional ubiquitin chains. Nat Struct Mol Biol. 2011;18:520-8.

3. Mevissen TET, Komander D. Mechanisms of Deubiquitinase specificity and regulation. Annu Rev Biochem. 2017;86:159-92.

4. Nicholson B, Marblestone JG, Butt TR, Mattern MR. Deubiquitinating enzymes as novel anticancer targets. Future Oncol. 2007:3:191-9.

5. Georges A, Marcon E, Greenblatt J, Frappier L. Identification and characterization of USP7 targets in Cancer cells. Sci Rep. 2018:8:15833.

6. Yeasmin Khusbu F, Chen FZ, Chen HC. Targeting ubiquitin specific protease 7 in cancer: a deubiquitinase with great prospects. Cell Biochem Funct. 2018;36:244-54

7. Lee JT, Gu W. The multiple levels of regulation by p53 ubiquitination. Cell Death Differ. 2010;17:86-92.

8. Kon N, Kobayashi Y, Li M, Brooks CL, Ludwig T, Gu W. Inactivation of HAUSP in vivo modulates p53 function. Oncogene. 2010;29:1270-9.

9. Hu M, Gu L, Li M, Jeffrey PD, Gu W, Shi Y. Structural basis of competitive recognition of p53 and MDM2 by HAUSP/USP7: implications for the regulation of the p53-MDM2 pathway. PLoS Biol. 2006;4:e27.

10. Kon N, Zhong J, Kobayashi Y, Li M, Szabolcs M, Ludwig T, et al. Roles of HAUSP-mediated p53 regulation in central nervous system development. Cell Death Differ. 2011;18:1366-75.

11. Zhang C, Lu J, Zhang QW, Zhao W, Guo JH, Liu SL, et al. USP7 promotes cell proliferation through the stabilization of Ki-67 protein in non-small cell lung cancer cells. Int J Biochem Cell Biol. 2016;79:209-21.

12. Meulmeester E, Maurice MM, Boutell C, Teunisse AF, Ovaa H, Abraham TE, et al. Loss of HAUSP-mediated deubiquitination contributes to DNA damageinduced destabilization of Hdmx and Hdm2. Mol Cell. 2005:18:565-76.

13. Cheng J, Yang H, Fang J, Ma L, Gong R, Wang P, et al. Molecular mechanism for USP7-mediated DNMT1 stabilization by acetylation. Nat Commun. 2015;6:7023.

14. Liang L, Peng $Y$, Zhang J, Zhang $Y$, Roy M, Han X, et al. Deubiquitylase USP7 regulates human terminal erythroid differentiation by stabilizing GATA1. Haematologica. 2019;2018:206227.

15. Tavana O, Li D, Dai C, Lopez G, Banerjee D, Kon N, et al. HAUSP deubiquitinates and stabilizes N-Myc in neuroblastoma. Nat Med. 2016;22: $1180-6$

16. Ma H, Chen H, Guo X, Wang Z, Sowa ME, Zheng L, et al. M phase phosphorylation of the epigenetic regulator UHRF1 regulates its physical association with the deubiquitylase USP7 and stability. Proc Natl Acad Sci U S A. 2012:109:4828-33

17. Wang Z, Kang W, You Y, Pang J, Ren H, Suo Z, et al. USP7: novel drug target in Cancer therapy. Front Pharmacol. 2019:10:427.

18. Zhou J, Wang J, Chen C, Yuan H, Wen X, Sun H. USP7: target validation and drug discovery for Cancer therapy. Med Chem. 2018;14:3-18.

19. Fu C, Zhu X, Xu P, Li Y. Pharmacological inhibition of USP7 promotes antitumor immunity and contributes to colon cancer therapy. OncoTargets and therapy. 2019;12:609-17.

20. Wang F, Wang L, Wu J, Sokirniy I, Nguyen P, Bregnard T, et al. Active sitetargeted covalent irreversible inhibitors of USP7 impair the functions of Foxp3+ T-regulatory cells by promoting ubiquitination of Tip60. PLoS One. 2017:12:e0189744.

21. Su D, Ma S, Shan L, Wang Y, Wang Y, Cao C, et al. Ubiquitin-specific protease 7 sustains DNA damage response and promotes cervical carcinogenesis. J Clin Invest. 2018;128:4280-96.

22. Yao Y, Zhang Y, Shi M, Sun Y, Chen C, Niu M, et al. Blockade of deubiquitinase USP7 overcomes bortezomib resistance by suppressing NFkappaB signaling pathway in multiple myeloma. J Leukoc Biol. 2018;104: 1105-15. 
23. Franqui-Machin R, Hao M, Bai H, Gu Z, Zhan X, Habelhah H, et al. Destabilizing NEK2 overcomes resistance to proteasome inhibition in multiple myeloma. J Clin Invest. 2018;128:2877-93.

24. Agathanggelou A, Smith E, Davies NJ, Kwok M, Zlatanou A, Oldreive CE, et al. USP7 inhibition alters homologous recombination repair and targets CLL cells independently of ATM/p53 functional status. Blood. 2017;130:156-66.

25. He ZL, Zheng H, Lin H, Miao XY, Zhong DW. Overexpression of polo-like kinase1 predicts a poor prognosis in hepatocellular carcinoma patients. World J Gastroenterol. 2009;15:4177-82.

26. Feng $Y$, Lin J, Liu Y, Tang Y, Zhou Y, Zhong M. Investigation of expressions of PDK1, PLK1 and c-Myc in diffuse large B-cell lymphoma. Int J Exp Pathol. 2019;100:32-40.

27. Zhao L, Bode AM, Cao Y, Dong Z. Regulatory mechanisms and clinical perspectives of miRNA in tumor radiosensitivity. Carcinogenesis. 2012;33: $2220-7$.

28. Zhuo X, Guo X, Zhang X, Jing G, Wang Y, Chen Q, et al. Usp16 regulates kinetochore localization of PIk1 to promote proper chromosome alignment in mitosis. J Cell Biol. 2015;210:727-35.

29. Daubeuf S, Singh D, Tan Y, Liu H, Federoff HJ, Bowers WJ, et al. HSV ICPO recruits USP7 to modulate TLR-mediated innate response. Blood. 2009;113: 3264-75.

30. An T, Gong Y, Li X, Kong L, Ma P, Gong L, et al. USP7 inhibitor P5091 inhibits Wnt signaling and colorectal tumor growth. Biochem Pharmacol. 2017:131:29-39.

31. Wang $M$, Zhang $Y$, Wang $T$, Zhang J, Zhou Z, Sun $Y$, et al. The USP7 inhibitor P5091 induces cell death in ovarian cancers with different P53 status. Cell Physiol Biochem. 2017;43:1755-66.

32. Weathington NM, Mallampalli RK. Emerging therapies targeting the ubiquitin proteasome system in cancer. J Clin Invest. 2014;124:6-12.

33. Gheghiani L, Loew D, Lombard B, Mansfeld J, Gavet O. PLK1 activation in late G2 sets up commitment to mitosis. Cell Rep. 2017;19:2060-73.

34. Combes G, Alharbi I, Braga LG, Elowe S. Playing polo during mitosis: PLK1 takes the lead. Oncogene. 2017;36:4819-27.

\section{Publisher's Note}

Springer Nature remains neutral with regard to jurisdictional claims in published maps and institutional affiliations.

Ready to submit your research? Choose BMC and benefit from:

- fast, convenient online submission

- thorough peer review by experienced researchers in your field

- rapid publication on acceptance

- support for research data, including large and complex data types

- gold Open Access which fosters wider collaboration and increased citations

- maximum visibility for your research: over $100 \mathrm{M}$ website views per year

At $\mathrm{BMC}$, research is always in progress.

Learn more biomedcentral.com/submissions 